Progress in Flight Physics 5 (2013) 571-582

DOI: 10.1051 /eucass/201305571

(C) Owned by the authors, published by EDP Sciences, 2013

\title{
ON OPTIMAL CONFIGURATIONS IN SUPERSONIC FLOW
}

\author{
V.I. Lapygin ${ }^{1}$, T. V. Sazonova ${ }^{1}$, and G. E. Yakunina ${ }^{2}$ \\ ${ }^{1}$ Central Research Institute of Machine Building (TSNIIMASH) \\ 4 Pionerskaya Str., Korolev, Moscow Region 141070, Russia \\ ${ }^{2}$ State University of Management \\ 99 Ryazansky Prosp., Moscow 109542, Russia
}

\begin{abstract}
Analytical solutions for variational problems on configurations of threedimensional (3D) bodies with the maximal lift-to-drag ratio at a given base area or a planform area are found within the limits of a localised interaction between the supersonic flow and the body surface. Functionals of considered variational problems depend on derivatives of the desired function with respect to independent variables only, and this simplifies the solution and allows studying the structure of the extremal surface. It is shown that the lower surface of optimal bodies is planar. If a base area is given, the upper surface is cylindrical with the generating line parallel to the oncoming flow velocity vector. If a planform area is given, the optimal body is a flat plate with the highest possible value of the liftto-drag ratio at a prescribed Mach number and friction coefficient. The optimal body with a planar upper surface is a wedge. These results are valid if the base pressure is taken into account and also for zero base pressure.
\end{abstract}

\section{INTRODUCTION}

Solutions of variational problems on aerodynamics of 3D configurations are based on simplified models of the supersonic flow interaction with a body. The local methods with the pressure coefficient defined by the angle between the normal to the body surface and the velocity vector of oncoming flow are examples of such models. Using the local methods there were found analytical solutions and examined configurations of planar, axisymmetric, and 3D bodies of the minimal drag [1] and, within the limits of slender bodies, of the maximal lift-to-drag ratio $[2-5]$.

Construction of a solution of the variational problem about configuration of a $3 \mathrm{D}$ body is connected with integration of partial differential equations with 
unknown function of two variables. However, sometimes the functionals for certain variational problems on the optimal configuration have the following form:

$$
\begin{gathered}
\Phi=\iint_{S} F(u, w) d x d y \\
y+f(x, z)=0 ; \quad u=\frac{\partial f}{\partial x} ; \quad w=\frac{\partial f}{\partial y} .
\end{gathered}
$$

Extremals of the functional $\Phi$ are defined by the following system:

$$
\frac{\partial F}{\partial u}=0 ; \quad \frac{\partial F}{\partial w}=0
$$

whose solutions are $u_{i}=$ const and $w_{i}=$ const. In other words, the extremals are the planar surfaces

$$
y+u_{i} x+w_{i} z+c_{i}=0 .
$$

In particular, considering the problem about the configuration of the minimal drag $C x_{\text {min }}$, it is easy to see that, with a given base area, the functional to be optimized has the following form:

$$
\Phi=\iint_{S} F(\alpha) d y d z
$$

where $\alpha=\left(1+u^{2}+w^{2}\right)^{-1 / 2}$, and the solutions are the surfaces satisfying the condition

$$
\alpha=\alpha^{*}=\text { conts },
$$

where $\alpha^{*}$ corresponds to the minimum of the function $F(\alpha)$ at a segment $[0,1]$.

This condition is satisfied by circular cone surfaces with a semivertex angle $\beta^{*}=\arcsin \left(\alpha^{*}\right)$ and planes, tangent to this cone. Infinite set of optimal bodies with a single value $C x_{\text {min }}$ could be constructed combining the segments of these surfaces [6-8]. The examples of optimal 3D bodies are illustrated in Fig. 1.
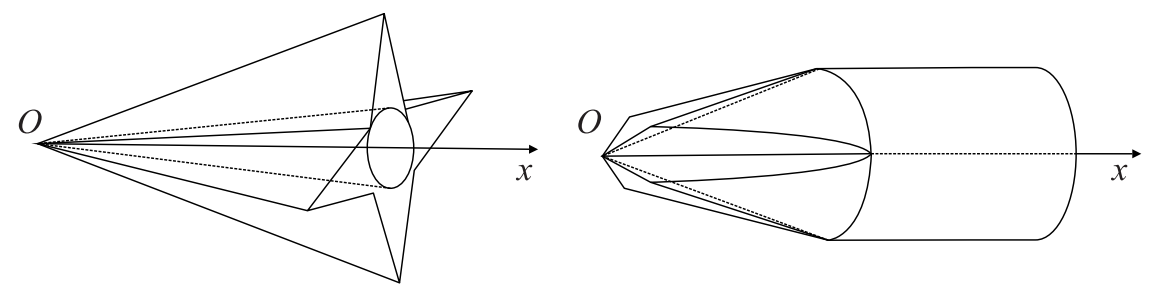

Figure 1 Examples of optimal 3D bodies 


\section{OPTIMAL CONFIGURATION OF THE GIVEN BASE SHAPE}

\subsection{Problem Statement}

Suppose that the velocity vector $\vec{v}$ of oncoming supersonic flow is parallel to $X$-axis of the body-axis Cartesian right system of coordinates $O X Y Z, \vec{v}=-\vec{x}$, where $\vec{x}$ is the unit vector of the $X$-axis. The $Y$-axis is pointing upwards. Let consider the bodies with a planar base situated in $Y O Z$ plane and the condition $\alpha=(\vec{n}, \vec{v})>0$ satisfied on the surface $x=f(y, z)$. Here, $\vec{n}$ is the unit vector of internal normal to a body surface element, and the pressure coefficient $C_{p}$ depends on $\alpha$ and Mach number M. Suppose also that friction coefficient $C_{f}$ is constant on the body surface, and the tangential stress vector lies in the plane of vectors $\vec{n}$ and $\vec{v}$.

Lift and drag aerodynamic coefficients are written in the following form:

$$
\begin{aligned}
S_{b} C_{y} & =\iint\left[C_{p}(\alpha)-\frac{C_{f} \alpha}{g}\right] u d y d z ; \\
S_{b} C_{x} & =\iint\left[C_{p}(\alpha)+\frac{C_{f} g}{\alpha}\right] d y d z
\end{aligned}
$$

where

$$
\begin{aligned}
S_{b} & =\iint d y d z ; \quad \alpha=\left(1+u^{2}+w^{2}\right)^{-1 / 2} ; \\
u & =\frac{\partial f}{\partial y} ; \quad w=\frac{\partial f}{\partial z} ; \quad g=\left(1-\alpha^{2}\right)^{1 / 2} .
\end{aligned}
$$

Integration is taken over the body base area $S_{b}$.

The lift-to-drag ratio is defined by the formula

$$
K=\frac{C_{y}}{C_{x}}
$$

and the problem is to find the function $f(y, z)$, which realizes the maximum of the functional (1) with the given area $S_{b}$. The first variation of the functional (1) is $\delta K=\left(\delta C_{y}-K \delta C_{x}\right) / C_{x}$; therefore, as follows from the condition $\delta K=0$, the problem is to find extremum of the functional

$$
\Phi=\iint F(\alpha, u) d y d z
$$

Here,

$$
F(\alpha, u)=\left[C_{p}(\alpha)-\frac{C_{f} \alpha}{g}\right] u-K\left[C_{p}(\alpha)+\frac{C_{f} g}{\alpha}\right]+\lambda
$$

where $\lambda$ is the constant Lagrangian coefficient; and $\alpha, g$, and $u$ are the functions of variables $y$ and $z$. 


\subsection{Analysis of Extremal Surfaces}

The equations of extremal surfaces are defined by the system:

$$
\frac{\partial F}{\partial u}=0 ; \quad \frac{\partial F}{\partial w}=0
$$

which has two families of solutions [9]:

$$
\begin{gathered}
w=0, C_{p}(\alpha)-\alpha g^{2} C_{p}^{\prime}(\alpha)-K\left[C_{f}-\alpha^{2} g C_{p}^{\prime}(\alpha)\right] \operatorname{sign}(u)=0 ; \\
u\left[C_{p}^{\prime}(\alpha)-\frac{C_{f}}{g^{3}}\right]-K\left[C_{p}^{\prime}(\alpha)-\frac{C_{f}}{\alpha^{2} g}\right]=0, C_{p}(\alpha)=\frac{C_{f} \alpha}{g}
\end{gathered}
$$

where

$$
C_{p}^{\prime}(\alpha)=\frac{d C_{p}(\alpha)}{d \alpha}
$$

The solution (2) defines a set of planes parallel to $Z$-axis:

$$
x+u_{1} y+c_{1}=0, \quad c_{1}=\text { const } .
$$

The solution (3) defines a set of planes (symmetrical respectively to the plane $X O Y$ ) that do not create a lift:

$$
x+u_{2} y+w_{2} y+c_{2}=0 ; \quad w_{2}= \pm \frac{\left[1-\alpha_{2}^{2}\left(1+u_{2}^{2}\right)\right]^{1 / 2}}{\alpha_{2}} ; \quad c_{2}=\text { const } .
$$

The optimal body should be formed by segments of the planes (2) and (3) so that the lower surface is parallel to $Z$-axis and is situated at the angle of attack $\beta_{1}=\arcsin \left(\alpha_{1}\right)$ to the flow. The upper surface is formed by two symmetrical planes (3).

The analysis of deduced extremal surface has shown that the $K$ maximum is realized at $\alpha_{2} \rightarrow 0$, and the formulation of the problem should include the surfaces with $\alpha=0[9]$.

\subsection{Construction of a Solution}

The lift-to-drag ratio is determined by the expression

$$
K=\frac{C_{y}}{C_{x}+C_{f} \Delta_{0}}, \quad \Delta_{0}=\frac{S_{0}}{S_{b}}
$$

where $C_{y}$ and $C_{x}$ are the lift and drag coefficients of a body with $\alpha>0 ; S_{0}$ is the area of body surface with $\alpha=0$. 
The condition of the functional (4) extremum is that the first variation vanishes:

$$
\delta K=\delta\left(C_{y}-K C_{x}\right)-K C_{f} \delta \Delta_{0}=0 .
$$

It follows that the section of body surface with $\alpha>0$ is the plane (2).

The section with $\alpha=0$ is a cylindrical surface, which generating line equation is the functional $\Delta_{0}$ extremal.

Let the body span to be $l=2 z_{k}$. The upper body surface with $\alpha=0$ may consist of three sections: one curvilinear section and two planar sections situated symmetrically respectively to $Y O X$ plane and parallel to it.

The area of curvilinear section is determined by the integral:

$$
S_{01}=2 u_{1} \int_{0}^{z_{k}} y\left(1+y^{\prime 2}\right)^{1 / 2} d z
$$

where $y^{\prime}=d y / d z, y(z)$ is the projection of curvilinear section at $Y O Z$ plane.

The area of the sections parallel to $Y O X$ :

$$
S_{02}=u_{1} y_{k}^{2}, \quad y_{k}=y\left(z_{k}\right) .
$$

The total area of the upper surface:

$$
S_{0}=S_{01}+S_{02}=u_{1} \delta_{0}, \quad \delta_{0}=2\left[\int_{0}^{z_{k}} y\left(1+y^{\prime 2}\right)^{1 / 2} d z+\frac{y_{k}^{2}}{2}\right] .
$$

Therefore, the problem is to find the minimum of the functional

$$
\Phi=\frac{y_{k}^{2}}{2}+\int_{0}^{z_{k}} y\left[\left(1+y^{2}\right)^{1 / 2}+\lambda_{1}\right] d z
$$

where $\lambda_{1}$ is the constant Lagrangian coefficient.

It follows from the analysis of the functional (5) extremals that if the coordinate $z_{k}$ is not given then the optimal body is a two-dimensional (2D) wedge with the upper surface parallel to the velocity vector $\vec{v}$.

If the body thickness $y(0)=y_{0}$ and area $S_{b}$ are given:

$$
y(z)=-t z+y_{0}, \quad t=\frac{y_{0}^{2}}{S_{b}}
$$

then the optimal body is a delta wing with a wedge-like profile. If the body span $l=2 z_{k}$ and a base area are given, then the base contour is determined by the relations: 


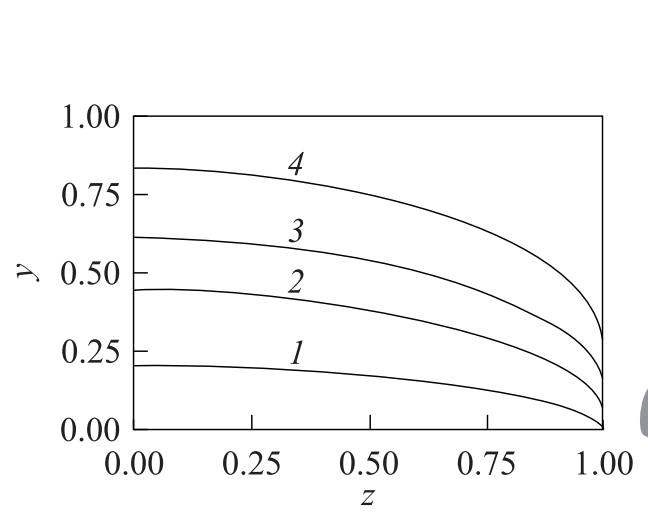

(a)

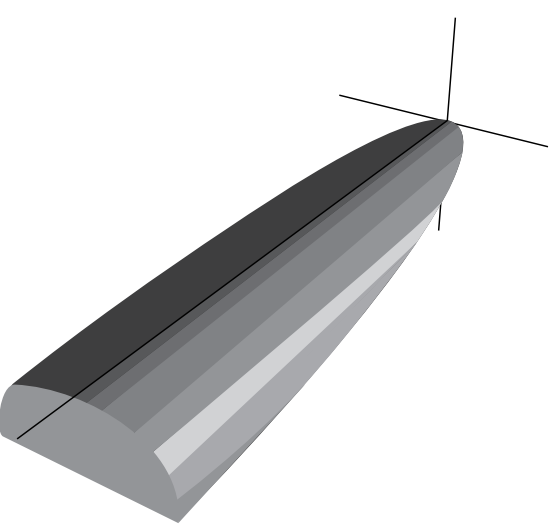

(b)

Figure 2 Optimal contours of the base area $(a)$ and 3D view of the optimal body $(b)$

$$
\left.\begin{array}{c}
z=\frac{\lambda_{1}\left(a y^{2}+b y+c\right)^{1 / 2}+c_{1} I}{a} ; \\
a=1-\lambda_{2}^{2} ; \quad b=\frac{2 \lambda_{1} a}{1+I^{*}} ; c=-\frac{a^{2}}{1+I^{*}} ; c_{1}=-\frac{c}{a} ; \\
I^{*}=|a|^{-1 / 2}\left[\frac{\pi}{2}+\arcsin \left(\left|\lambda_{1}\right|^{-1}\right)\right] ; \\
I=|a|^{-1 / 2}\left[\frac{\pi}{2}+\arcsin \left(\lambda_{1} \mid-\left(1+I^{*}\right) y\right)\right] .
\end{array}\right\}
$$

Here, all linear dimensions are given in the ratio to $z_{k}$.

Optimal contours of the base area are presented in Fig. $2 a$ with different $\Delta_{k}=S_{b} /\left(2 z_{k}^{2}\right)$; a $3 \mathrm{D}$ view of the optimal body is illustrated in Fig. $2 b$. The body base shape does not depend on $u_{1}, \mathrm{M}, C_{f}$, and, consequently, on specific form of the dependence $C_{p}(\alpha)$.

Let determine the angle of attack $\beta_{1}$ and the maximal lift-to-drag ratio at $\gamma$ $=1.4$, using the local wedge formula [8]:

$$
C_{p}(\alpha)=\frac{\gamma+1}{2}\left[1+\left(1+\left(\frac{4}{(\gamma+1) \alpha \sqrt{\mathrm{M}^{2}-1}}\right)^{2}\right)^{1 / 2}\right]
$$

The values of $\beta_{1}^{*}$ and $K^{*}$ for the optimal 2D wedge at $\gamma=1.4$ are presented in Table 1 (the left numbers in the cells).

For the bodies defined by the formulas (6), the optimal angles $\beta_{1}$ and the maximal lift-to-drag ratios $K$ are plotted against $\Delta_{k}$ in Fig. 3 for $C_{f}=0.002$ and $\mathrm{M}=6$ and 10 . Note that the $\beta_{1}$ values are close to the $\beta_{1}^{*}\left(\beta_{1}-\beta_{1}^{*}<30^{\prime}\right)$. 
Table 1 The values of $\beta_{1}^{*}$ and $K^{*}$ for the optimal 2D wedge at $\gamma=1.4$

\begin{tabular}{|c|c|c|c|c|c|c|}
\hline \multirow{3}{*}{$\mathrm{M}$} & \multicolumn{6}{|c|}{$C_{f}$} \\
\hline & \multicolumn{2}{|c|}{0.001} & \multicolumn{2}{|c|}{0.002} & \multicolumn{2}{|c|}{0.003} \\
\hline & $\beta_{1}^{*}$ & $K^{*}$ & $\beta_{1}^{*}$ & $K^{*}$ & $\beta_{1}^{*}$ & $K^{*}$ \\
\hline 6 & $4.32^{\circ} / 3.36^{\circ}$ & $7.34 / 9.28$ & $6.00^{\circ} / 4.84^{\circ}$ & $5.42 / 6.62$ & $7.24^{\circ} / 6.00^{\circ}$ & $4.55 / 5.45$ \\
\hline 10 & $5.32^{\circ} / 4.50^{\circ}$ & $6.37 / 7.44$ & $7.19^{\circ} / 6.34^{\circ}$ & $4.83 / 5.43$ & $8.49^{\circ} / 7.68^{\circ}$ & $4.13 / 4.55$ \\
\hline 15 & $5.96^{\circ} / 5.41^{\circ}$ & $5.96 / 6.54$ & $7.82^{\circ} / 7.33^{\circ}$ & $4.61 / 4.91$ & $9.11^{\circ} / 8.71^{\circ}$ & $3.97 / 4.18$ \\
\hline$\infty$ & $6.75^{\circ} / 6.75^{\circ}$ & $5.59 / 5.59$ & $8.50^{\circ} / 8.50^{\circ}$ & $4.41 / 4.41$ & $9.72^{\circ} / 9.72^{\circ}$ & $3.84 / 3.84$ \\
\hline
\end{tabular}

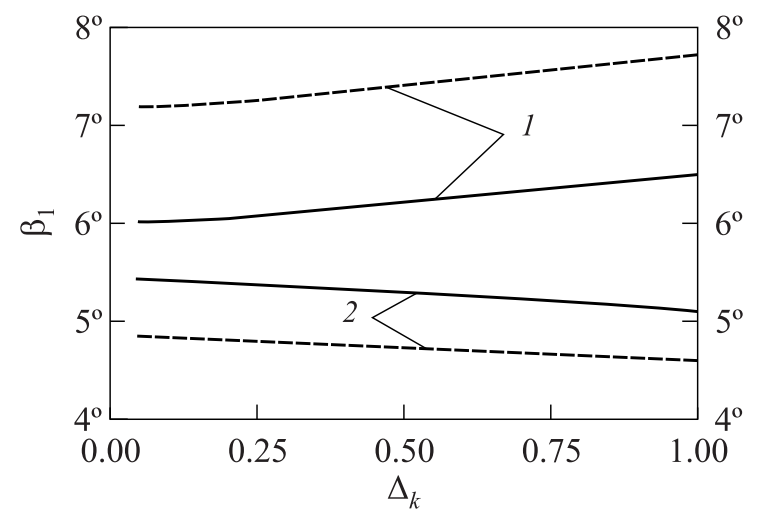

Figure 3 The optimal angles $\beta_{1}(1)$ and the maximal lift-to-drag ratios $K(2)$ vs. $\Delta_{k}=S_{b} /\left(2 z_{k}^{2}\right)=\int_{y_{0}}^{y_{k}} z d y+y_{k}$ for $C_{f}=0.002$ and $\mathrm{M}=6$ (solid curves) and 10 (dashed curves)

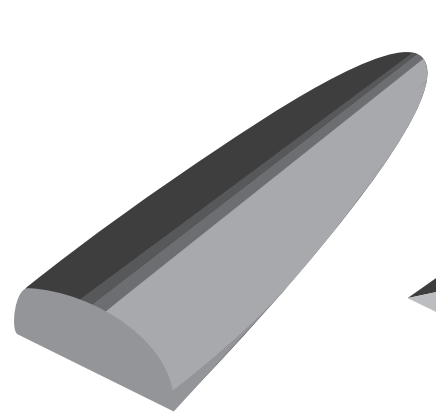

(a)

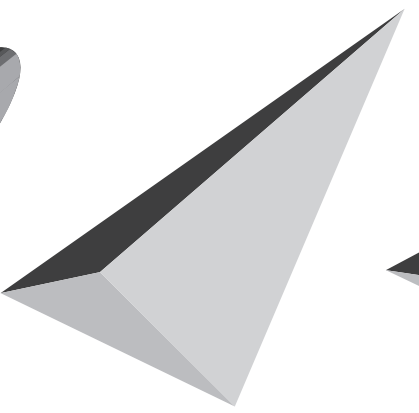

(b)

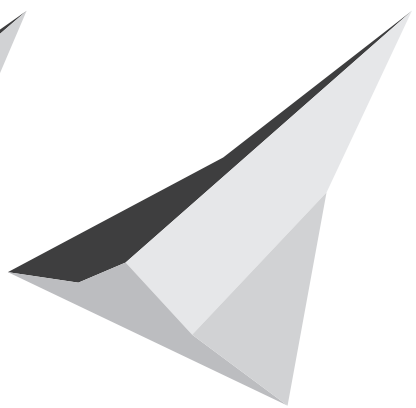

(c)

Figure 4 Configurations of the optimal bodies with various base shapes at $S_{b} / y_{0}^{2}$ $=\pi / 2, \mathrm{M}=15$, and $C_{f}=0.002:(a)\left(\beta_{1}, K\right)=\left(8.21^{\circ}, 4.40\right) ;(b)\left(8.09^{\circ}, 4.46\right) ;$ and (c) $\left(\beta_{1}, K\right)=\left(8.1^{\circ}, 4.45\right)$ 
The values of the lift-to-drag ratio of optimal bodies with a triangular and a curvilinear base with a similar area $S_{b}$ and the length $L$ are close. At the same time, the span of the optimal delta wing is 1.5 times greater than the span of a body defined by the relations (6).

If the base contour is given, the curve $y=y(z)$ makes a guiding line of the upper cylindrical surface with generating lines parallel to $X$-axis. The configurations of the optimal bodies with various base shape are illustrated in Fig. 4 at $S_{b} / y_{o}^{2}=\pi / 2, \mathrm{M}=15$, and $C_{f}=0.002$. The corresponding pairs of $\left(\beta_{1}, K\right)$ values are: $(a)\left(8.21^{\circ}, 4.40\right) ;(b)\left(8.09^{\circ}, 4.46\right)$; and $(c)\left(8.1^{\circ}, 4.45\right)$.

\section{OPTIMAL CONFIGURATION OF THE GIVEN PLANFORM AREA}

Let consider the bodies with a depression flow realized at a part of their surface. The equation for the lower body surface takes form: $y+f_{1}(x, z)=0$, and for the upper surface: $y+f_{2}(x, z)=0$. Here, at the lower surface, the pressure coefficient $C_{p 1}>0$, and at the upper surface $C_{p 2} \leq 0$. With assumptions accepted in the previous section, the aerodynamic coefficients are determined by the formulas:

$$
\begin{gathered}
S_{b} C_{y}=\iint\left(\frac{C_{p 1}-C_{p 2}-C_{f}}{\Delta_{11}}-\frac{C_{f}}{\Delta_{21}}\right) d x d z ; \\
S_{b} C_{x}=\iint\left(C_{p 1} u_{1}-C_{p 2} u_{2}+C_{f} \Delta_{11}+C_{f} \Delta_{21}\right) d x d z ; \\
K=\frac{C_{y}}{C_{x}} ; \quad S_{b}=\iint d x d z .
\end{gathered}
$$

The integration is taken over a section in $X O Z$ plane corresponding to the body planform area:

$$
\begin{array}{rlrl}
u_{i} & =\frac{\partial f_{i}}{\partial x} ; & w_{i} & =\frac{\partial f_{i}}{\partial z} ; \\
\Delta_{i}^{2} & =1+u_{i}^{2}+w_{i}^{2} ; \Delta_{i 1}^{2} & =1+w_{i}^{2} ; \\
(\vec{n} \cdot \vec{v}) & =\frac{u_{i}}{\Delta_{i}} ; & C_{p i} & =C_{p}\left(\frac{u_{i}}{\Delta_{i}}\right), i=1,2 .
\end{array}
$$

The determination of a configuration of the maximum lift-to-drag ratio is reduced to finding the minimum of the functional

$$
\begin{aligned}
& \Phi=\iint F\left(u_{i}, w_{i}\right) d x d z \\
& F=C_{p 1}-C_{p 2}-\frac{C_{f}}{\Delta_{11}}-\frac{C_{f}}{\Delta_{21}}-K\left(C_{p 1}-C_{p 2}+C_{f} \Delta_{11}+C_{f} \Delta_{21}\right)+\lambda_{2}
\end{aligned}
$$

where $\lambda_{2}$ is the constant Lagrangian coefficient. 
Extremal surfaces are defined by the system of equations:

$$
\frac{\partial F}{\partial u_{i}}=0, \quad \frac{\partial F}{\partial w_{i}}=0, i=1,2 .
$$

For the upper and lower surfaces, these equations have two families of solutions for each surface. The first family for the lower surface defines planar surfaces parallel to $Z$-axis:

$$
w_{1}=0 ; \quad C_{p 1}^{\prime} \frac{1}{\Delta_{1}^{3}}-K\left(C_{p 1}^{\prime} \frac{1}{\Delta_{1}^{3}}+C_{p 1}\right)=0 .
$$

The second family is defined by the equations:

$$
\left.\begin{array}{r}
C_{p 1} u_{1}+C_{f} \Delta_{11}=0 ; \\
C_{p 1}^{\prime} \frac{\Delta_{11}^{2}}{\Delta_{1}^{3}}-K\left(C_{p}^{\prime} \frac{\Delta_{11} u_{1}}{\Delta_{1}^{3}}+C_{p 1}+C_{p 1} \frac{u_{1}^{2}}{\Delta_{1}^{2}}+C_{f} \frac{\Delta_{11} u_{1}}{\Delta_{1}^{2}}\right)=0 .
\end{array}\right\}
$$

The first equation in (9) conflicts with the condition $C_{p 1}>0$ and corresponds to zero drag of the lower surface that has no physical meaning. Consequently, the lower surface of the optimal body is a plane parallel to $Z$-axis. The angle of attack for this plane $\beta_{1}=\operatorname{arctg} u_{1}$ is determined from the second equation in (8). It can be shown by analogy that the upper body surface is also a surface parallel to $Z$-axis $\left(w_{2}=0\right)$. Therefore, the upper and lower surfaces of the body are the planar surfaces, and the optimal body is a flat plate with the angle of attack $\beta_{1}$.

In fact, the analyzed problem statement does not include the planes parallel to $Y$-axis, which add nonzero thickness to the optimal body. But such planes make the drag greater and do not influence upon the lift; so, they reduce the lift-to-drag ratio $K$. That is why such planes cannot be the extremals.

Let analyze two limiting cases of discussed problem solutions:

(1) $\beta_{1}\left(\mathrm{M}^{2}-1\right)^{1 / 2} \ll 1$; and

(2) $\sin \beta_{1}\left(\mathrm{M}^{2}-1\right)^{1 / 2} \gg 1$.

For the first case,

$$
C_{p 1}=-C_{p 2}=\frac{2 \beta_{1}}{\sqrt{\mathrm{M}^{2}-1}}, \quad \beta_{1}=\beta_{2} .
$$

For the second case, according to (7),

$$
C_{p 1}=(\gamma+1) \frac{u_{1}^{2}}{\Delta_{1}^{2}} ; \quad C_{p 2}=0 .
$$


The second equation in (8) for the upper surface becomes an identical relation valid with arbitrary $u-2>0$. Therefore, in this case, $u_{1}=u_{2}$ too. The correctness of the condition $u_{1}=u_{2}$ for the finite values of Mach number was checked by a numerical optimization of the bodies of various planforms with the help of the code presented in [10]. Optimal values for $\beta_{1}$ and $K$ of a flat plate are presented in Table 1 (the right numbers in cells); it is seen that at moderate Mach numbers, the flat plate has lesser angles of attack $\beta_{1}$ and greater $K$ values compared to a $2 \mathrm{D}$ wedge.

\section{Optimal Configuration of Given Planform Area and Planar Upper Surface}

Let examine a body, whose upper surface coincides with $X O Z$ plane, and its length equals 1 . In accordance with the above analysis, the lower surface is planar. Obviously, for constructing the closed body surface it is necessary to introduce a cylindrical surface with generating lines parallel to $Y$-axis. For this purpose consider a body formed by two planes $y=0$ and $y=u_{1} x$ and the cylindrical surface $z=f(x)$. Finding of a configuration of the maximal lift-todrag ratio with known $u_{1}$ and given planform area $S_{b}$ is reduced to searching the extremal of the functional

$$
\begin{aligned}
\Phi_{1} & =\int F\left(x, z, z^{\prime}\right) d x \\
F & =\left(\frac{2 z^{\prime 3}}{1+z^{\prime 2}}+C_{f} \sqrt{1+z^{\prime 2}}\right) x+\lambda z .
\end{aligned}
$$

The equations for the extremal and boundary conditions are written in the following form:

$$
\lambda+\frac{d}{d x} F_{z^{\prime}}=0 ; \quad F_{z^{\prime}}=0 \text { at } x=0,1 .
$$

It follows that $\lambda=0$ and the extremal is a straight line $z=c=S_{b} / 2$.

Thus, if the upper surface is planar, then the optimal body is a wedge. Obviously, the greater is the span, the greater is the $K$ value. It is noted that for all examined cases, this solution is true also if the base drag is taken into consideration on the condition that the pressure coefficient in the base region is constant $\left(C_{p b}=\right.$ const $)$.

\section{CONCLUDING REMARKS}

The result of the analysis with the assumption about a local character of the force interaction between the flow and the body surface are analytical solutions 
on configurations of the maximal lift-to-drag ratio at supersonic flow velocities. For a given base area or a given planform area, the lower (windward) surface of the optimal body is flat. This conclusion agrees with the research results on the influence of $\mathrm{V}$-shape upon a value of the lift-to-drag ratio of $\mathrm{V}$-shaped wings at supersonic Mach numbers [11].

The construction of solutions for variational problems was generated with minimal constraints on a body shape - either a base area or a planform area. Therefore, the values of the lift-to-drag ratio of optimal configurations are extremely accessible or upper bounds of the lift-to-drag ratio at supersonic velocities.

The analytical results do not conflict with numerical data on the investigation of configurations like waveriders $[12,13]$. This work is an additional argumentation in favour of the local approach, which allows, as well as for the problem on a body of the minimal drag [6-8], finding the solutions consistent with the solutions found with more accurate assumptions about the character of the force interaction between a flow and a body surface.

\section{ACKNOWLEDGMENTS}

The work is supported financially by Russian Foundation for Basic Researches (project 09-01-00171a).

\section{REFERENCES}

1. Miele, A., ed. 1965. Theory of optimum aerodynamic shapes. New York: Academic Press.

2. Maikapar, G. I. 1966. A wing with the maximum lift/drag ratio at supersonic velocity. J. Appl. Math. Mech. 30(1):227-31.

3. Miele, A., D. G. Hull, and S. L. Brown. 1967. Maximum lift-to-drag ratio of a slender flat-top hypersonic body. Astronautica Acta 13(2).

4. Huang, H. Y. 1968. Conical bodies of given length and volume having maximum lift-to-drag ratio at hypersonic speeds. Part 2: Variational methods. J. Astr. Sci. 15(3):118-23.

5. Lapygin, V. I., and D. M. Fofonov. 2006. Determination of limiting values for lift-todrag ratio in hypersonic flow. Cosmonautics Rocket Eng. 3(44):21-27. [In Russian.]

6. Yakunina, G. Ye. 2000. The construction of optimum three-dimensional shapes within the framework of a model of local interaction. J. Appl. Math. Mech. 64(2):289-98.

7. Yakunina, G. Ye. 2000. The optimum non-conical and asymmetrical threedimensional configurations. J. Appl. Math. Mech. 64(4):583-91. 
8. Kraiko, A. N., and G. E. Yakunina. 2008. Optimal body design using localized interaction models. J. Appl. Math. Mech. 72(1):26-32.

9. Lapygin, V.I., and G. Ye. Yakunina. 2009. The shapes of bodies with maximum lift-to-drag ratio in supersonic flow. J. Appl. Math. Mech. 73(5);514-23.

10. Lapygin, V. I., and D. M. Fofonov. 2009. Optimization of hypersonic flight vehicle configurations. Certificate of official registration of computer codes No. 2009612692.

11. Lapygin, V. I., and P. V. Tret'yakov. 1986. Conical wing of maximum lift-drag ratio in a supersonic gas flow. Fluid Dyn. 21(3):445-50.

12. Keldysh, V. V. 1996. Optimal shape of lifting bodies in a flow with a plane shock. Fluid Dyn. 31(4):588-96.

13. Mazhul, I. I. 2007. A comparative analysis of the aerodynamics of waveriders designed on the basis of conical and planar flows. Thermophys. Aeromechanics 14(1):93-105. 